\title{
"We've Not Gotten Even Close to What We Want to Do": a Qualitative Study of Early Patient-Centered Medical Home Implementation
}

\author{
Anaïs Tuepker, $P h D, M P H^{1,2}$, Devan Kansagara, $M D, M C R^{1,2}$, Eleni Skaperdas, $B A^{7}$, \\ Christina Nicolaidis, MD, MPH', 3,4 , Sandra Joos, $P h D^{1,2}$, Michael Alperin, MD, MBA ${ }^{7}$, and David \\ Hickam, MD, MPH', 2,5

\begin{abstract}
'Portland VA Medical Center, Health Services Research \& Development/ VISN 20 PACT Demonstration Laboratory, Veterans Health Administration, Portland, OR, USA; ${ }^{2}$ Division of General Internal Medicine and Geriatrics, School of Medicine, Oregon Health \& Science University, Portland, OR, USA; ${ }^{3}$ School of Social Work, Portland State University, Portland, OR, USA; ${ }^{4}$ Department of Public Health \& Washington, DC, USA.
\end{abstract} \\ Preventive Medicine, Oregon Health \& Science University, Portland, OR, USA; ${ }^{5}$ Patient Centered Outcomes Research Institute (PCORI),
}

BACKGROUND: The Veterans Health Administration (VA) Patient Aligned Care Team (PACT) initiative is designed to deliver a medical home model of care associated with better patient outcomes, but success will depend in part on the model's acceptability and sustainability among clinic employees.

OBJECTIVE: We sought to identify key themes in the experience of primary care providers, nurse care managers, clerical and clinical associates, and clinic administrators implementing PACT, with the aim of informing recommendations for continued development of the model and its components.

DESIGN: Observational qualitative study; data collection from 2010 to 2013 , using role-stratified and team focus groups and semi-structured interviews.

PARTICIPANTS: 241 of 337 (72\%) identified primary care clinic employees in PACT team or administrative roles, from 15 VA clinics in Oregon and Washington.

APPROACH: Data coded and analyzed using conventional content analysis techniques.

KEY RESULTS: Overall, participants were enthusiastic about the PACT concept, but felt necessary resources for success were not yet in place. Well-functioning teams were perceived as key to successful implementation. Development of such teams depended on adequate staffing, training, and dedicated time for team development. Changes within the broader VA system were also seen as necessary, including devolving greater control to the clinic level and improving system alignment with the PACT model. PACT advocates from among clinic and institutional level leadership were identified as a final key ingredient for success. These themes were consistent despite differences in clinic settings and characteristics.

CONCLUSIONS: PACT implementation faced significant challenges in its early years. Realizing PACT's transformative potential will require acting on the needs identified by clinic workers in this study: ensuring

Electronic supplementary material The online version of this article (doi:10.1007/s11606-013-2690-z) contains supplementary material, which is available to authorized users.

Published online April 9, 2014 adequate staffing in all team roles, devoting resources to in-depth training for all employees in communication and other skills needed to maximize team success, and aligning the broader VA hospital system with PACT's decentralized, team-based approach.

KEY WORDS: primary care redesign; patient-centered medical home; team-based care; Veterans; health services research; qualitative research.

J Gen Intern Med 29(Suppl 2):S614-22

DOI: $10.1007 / \mathrm{s} 11606-013-2690-z$

(c) Society of General Internal Medicine 2013

\section{INTRODUCTION}

The patient-centered medical home $(\mathrm{PCMH})$ has gained considerable traction as a proposed solution to persistent challenges facing US healthcare in general and primary care in particular. ${ }^{1-3}$ Defined by a focus on care that is teambased, data-informed, highly accessible, continuous, and coordinated across the spectrum of care, the PCMH is associated with positive outcomes for patients. ${ }^{4}$ Medical home features, such as enhanced patient access, improved scheduling, care provider continuity, and care coordination activities, have been associated with lower rates of avoidable hospitalizations and decreased Emergency Department use. ${ }^{5-8}$ Multiple difficulties in implementing the PCMH model, however, have been documented. The move away from a physician-centric model of care towards one that is truly team-oriented, and the challenges of aligning financial reimbursement with the PCMH's emphasis on proactive prevention, are only two of the model's persistent challenges across diverse primary care practices. ${ }^{9-11}$

In 2010, the Veterans Health Administration (VA) rolled out its version of the $\mathrm{PCMH}$, the Patient Aligned Care Teams (PACT) initiative. With an initial focus on primary care, PACT emphasizes common features of the PCMH 
model: team-based care for a defined patient panel, proactive population management, and improved continuity and coordination of care. According to the model, each care team consists of a Primary Care Provider (PCP), Nurse Care Manager (NCM), Clinical Associate (C/CA), and Clerical Associate $(\mathrm{C} / \mathrm{CA})$, and is further supported by social workers, pharmacists, nutritionists, and psychologists.

Despite this focus on the team as an important conduit for improving care delivery, there has been relatively little research investigating perceptions of facilitators and barriers to PACT implementation from the perspective of front-line employees in all roles. The VA setting affords an important opportunity to evaluate the challenges and opportunities inherent in implementing team-based care in a large, integrated healthcare system that includes clinics varying in their local settings and physical resources.

Our study objective was to understand the perspectives of VA primary care clinic employees on the actual implementation of the PACT model. Informed by the implementation science approach of the Consolidated Framework for Implementation Research, ${ }^{12}$ our analysis focused on themes with potential to aid effective wide-spread implementation of team-based primary care approaches.

\section{METHODS}

\section{Setting}

Our Demonstration Lab is evaluating PACT implementation in 15 primary care clinics within VA Integrated Service Network 20, using a mixed-methods approach that analyzes qualitative process data as well as longitudinal analysis of administrative data on patient service use and health outcomes. Participating clinics include hospital-based clinics as well as Community Based Outpatient Clinics (CBOCs), located in both urban and rural settings in Oregon and southern Washington. Clinic size varies, with a range of three to 48 Primary Care employees per clinic. Most data were collected 8-20 months after an official April 2010 roll-out of the PACT initiative; the last focus group was conducted in February 2013 at a clinic that opened in June 2012.

\section{Data collection}

We conducted focus groups and individual interviews with employees at 15 VA primary care clinics. All clinical and administrative employees were eligible to participate. Experienced facilitators and qualitative interviewers conducted all focus groups and interviews, which were audio recorded unless participants objected (three interviews) or institutional approval to record was not granted (one focus group). We interviewed clinic leadership, defined as Group Practice Managers (GPMs), who provide clinical leadership to the primary care providers, and Operations Managers, who supervise all other employees as well as providing administrative management. Four employees requested private interviews, which were conducted. In clinics with more than two teams or ten employees, we stratified focus groups by team role into PCPs, NCMs, and Clerical/Clinical Associates (C/CAs). We did this in order to promote open discussion about relationships across roles, which we anticipated might be a difficult subject. We used focus group and interview guides that asked about initial expectations of PACT, perceived barriers and facilitators to implementation, and general perceived strengths and weaknesses in clinics' current practices; these guides are available as online appendices. The Institutional Review Board of the Portland VA Medical Center approved this study.

\section{Data analysis}

We transcribed all recordings and interview notes. Two research assistants trained in conventional content analysis ${ }^{13}$ doublecoded the data using Atlas.ti7.0.91 software (Atlas.ti $\mathrm{GmbH}$, Berlin). In conventional content analysis, themes are iteratively developed as immersion in the data generates insights, and we thus revised our codebook throughout the first rounds of data analysis, keeping an audit trail of changes made. Two experienced qualitative researchers, one of whom is also a non-VA primary care provider, supervised the coding and led the analysis. Our coding approach included the use of memos to highlight dissenting or unusual observations, in order to ensure that such comments were not overlooked in analysis. Rapid analysis of results and feedback sessions presented to nine of the clinics served as a participant validity check and helped identify preliminary themes that were further refined during coding. The full investigative team met multiple times to discuss findings, collapse codes, and decide on a final thematic structure.

\section{RESULTS}

We conducted 32 focus groups and 21 semi-structured interviews with 241 employees from 15 primary care clinics. Participant characteristics are described in Table 1. Focus groups ranged from 29 to 60 minutes in length; interviews ranged from 16 to 43 minutes in length.

\section{Theme 1: Rift Between PACT Theory and Reality}

Overall, participants in all employee roles expressed enthusiasm about the PACT model and its key concepts. Many employees saw team-based care as a better way of working. Notably, clinical and clerical associates were often vocal about experiencing enhanced satisfaction in this model:

Before, I think all we were doing is just doing, just working and put[ting] the patient in. But now that we're on a team I feel like we're really focusing on 


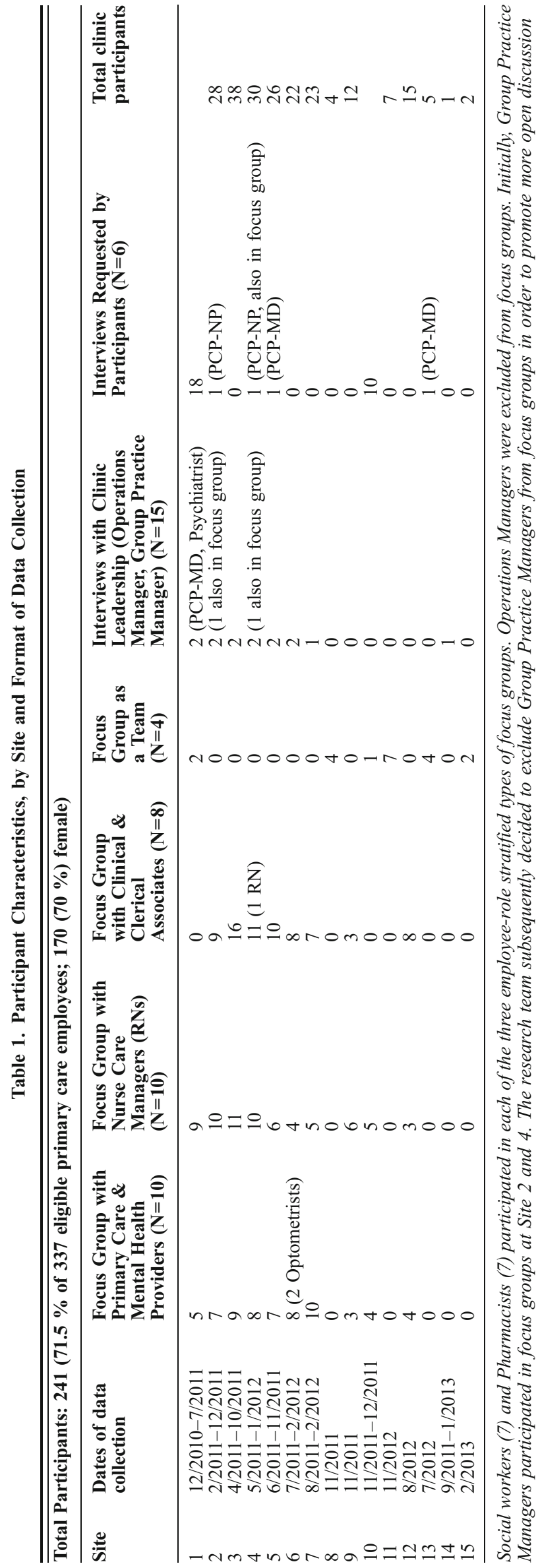

doing better care... we're really focusing on doing preventative stuff and making the patients healthier. By condensing it to that team, for me it's easier. [C/CA] It's nice to be able to work with a team, and be able to manage a set of patients, and they're my patients that, you know, I'm taking care of, and I get to establish a relationship with them. ...it's really nice to work for an organization that values nursing and values this model. And even though there are things that need to be fixed, it's nice that that is the goal. [C/CA]

This enthusiasm was tempered, however, by the perception that clinics were missing key resources necessary for full, sustainable implementation. As one person stated, "It's like we understand what the foundation is to build this medical home, and we've been given the blueprints, but we haven't been given the tools that we need to implement it, to get it going. It's stressful." A few employees in all roles shared one RN's stated attitude of "ok, let's just wait this one out," as they watched for signs of long-term organizational commitment to the transformation, such as hiring additional employees, funding and making time for PACT training opportunities, and allowing scheduled time for population management. These actions emerged as key to convincing employees that the model was here to stay.

\section{Theme 2: Creating a Well-Functioning Team Comes First, Faces Challenges}

Across clinics and roles, two themes consistently intertwined: the importance of a well-functioning team, and the impact of staffing constraints on team development. A well-functioning team was widely seen as crucial to being able to implement the PACT approach. PACT team characteristics differed from those of the more general clinic-level "team," where everyone helps out as needed. Employees described a well-functioning PACT team as one whose members 1 ) regularly and frequently communicate openly and honestly with each other; 2) know and trust each other's abilities; 3 ) have clear roles; 4) are comfortable with delegation and task-sharing; and 5) can rely on each other's regular physical presence on a day-to-day basis.

Team function was frequently mentioned in relation to the core PACT activity of population management. With a team, population management was both possible and rewarding.

Once we started the team and I was on a team that was fully staffed, it was really exciting for me because we really focused on our... 1,200 patients, so we started looking at the LDLs and A1Cs and we broke down every single patient and who had 'em, then we worked on getting them off, lowering them. [C/CA] 
In this context, participants often felt that inadequate staffing posed an insurmountable barrier to solid team function. At the time of our data collection, in no clinic were all teams staffed to PACT recommendations. Many participants described simply not having enough people to cope with existing (or increasing) workloads. In larger clinics, covering for usual annual leave requirements would require several full-time "float" employees, yet current staffing models ignored this need. Inadequate staffing hindered teams' abilities to meet required process measures, deliver optimal patient care, and maintain positive clinic morale. The reliable, frequent communication between team members that participants saw as necessary to team function was described as routinely disrupted when employees had to support multiple teams simultaneously.

Most providers here would say the same thing, that my RN, my MA are usually too stretched thin in caring for some other $R N$ who is sick or gone or whatever the staffing problem is; we're not really focused as a team because their responsibilities are usually split in covering for another MA, another provider, and to do those responsibilities. So probably as many days as not, the team doesn't really exist, they're just trying to do their own jobs, and so am I. [PCP]

Right now I have two providers, and that's really hard for me to get into this PACT concept, because I'm busy with alerts from two providers, I can't get down like some of the other nurse care managers and really concentrate on the chronic disease management. So I'm just trying to stay alive with the alerts and walk-ins and, you know, appointments. But eventually, hopefully, we're training another person, so that will help. [NCM]

In clinics where staffing did increase over time, participants' support for PACT increased alongside their perception that the system was committed to supporting the change effort. Teambuilding, participants agreed, took time: time working together and learning to trust and know one another's abilities. As individual teams achieved stability, some clinics began to see progress, as one $\mathrm{CBOC}$ manager reflected seventeen months after PACT's official launch: "The first six, eight months it was just awful, but the last month is just like: wow. I mean it's just amazing what's happened in the last month since we got all the teams up and running."

\section{Theme 3: PACT Requires Greater Primary Care Control Within a Supportive, Well-Aligned System}

Participants had observed little change in the larger VA system, even though PACT was a high priority national initiative. As with staffing, participants interpreted the lack of larger systemic change as a barrier to implementation and an indication of ambivalent commitment.

I would say at the highest level leadership has behaved like people didn't believe in PACT. PACT is sort of a something they're trying to engraft on a system that otherwise hasn't changed how it does business. Human Resources is impossible. IT [Information Technology] is unresponsive and fragmented and non-supportive. So basically you have this model, but the VA hasn't changed fundamentally how it goes about business and in fact it is essentially starving the PACT model by continuing to do business in the old way in the rest of the organization. [PCP]

Employees wanted system change to allow clinics greater decision-making control than had historically been the case. Specific examples included being able to decide which PACT components to implement first, and having all patient appointments scheduled by teams, rather than by remote call centers. Employees in all roles felt strongly that clinic-level scheduling was the most appropriate approach within the PACT model and were frustrated by decisions to expand the use of call centers.

...there are different groups within the medical facility that can book into our clinics that have nothing to do with our team... they do not know the patient, and because they do not know the patient... they have really, truly no idea of what the needs for the patient are. And they often [could] substitute a face-to-face appointment with just good old-fashioned communication. [PCP]

We have some control, but I think there's more that we need to have control of. Like, I've asked several times to not have an 8:45 patient on Wednesday morning [when the PACT team meets]. And every week I have an 8:45 appointment on Wednesday morning. So it's like kind of, "here, you're in charge." But not really. [PCP]

The established practice of hiring a PCP to create a panel, with other team positions filled sometimes months later, was another frequently cited example of a system deaf to the needs of the PACT model, since it reinforced a "physician-first" hierarchy and minimized clinic participation in hiring decisions.

We need support staff. But the PACT model seems to be top heavy... we need the MAs and we need the LPNs in here. And we're not getting them. [C/CA]

Participants wanted to see the PACT model applied within specialty clinics in order to maximize the model's potential.

It seems like primary care is the first to go with most everything-secure messaging, PACT, answering all 
our calls live, but the rest of the institution doesn't start until later or sometimes never even gets there. And that creates barriers then for us to be efficient or effective as we could be. [Operations Manager]

PCPs in particular often noted the tension between the PACT model and their need to meet productivity and access goals that had not been changed to account for PACT priorities. Without PACT-aligned measures to capture team (not just PCP) workload and care coordination activities, many employees felt this indicated, at best, an oversight that made it hard to measure success, and, at worst, a trivialization of the team's contribution to outcomes.

\section{Theme 4: Training is Needed for Specific PACT Skills}

Participants emphasized the need for further training related to PACT. In the absence of such training, well-functioning teams only emerged through luck, when team members were unusually motivated or skilled. Several comments echoed one CBOC manager's reflection that "if I were to have to start over, I would probably start with even more team building." Others admitted an unexpected appreciation of trust and communication skills that were previously undervalued:

What I would prioritize for my team-...[and $] I$ can't believe I would ever say I would want to do, because it sounds like middle management hooey-but actually, trust-building exercises. Because...the biggest barrier in implementing a lot of those things is resistance to change. [PCP]

Clinical Associates in particular also identified the need for training in specific technical skills needed for PACT implementation. Text Box 1 lists some specific training suggestions.

\section{Text Box 1. Training needs identified by primary care staff participants}

- $\quad$ Tools for team building (TeamSTEPPS and others)

- Applied panel management skills

- Defining care management (to target Nurse Care Managers)

- Motivational Interviewing

- Patient-centered communication skills

- Basic training in Chronic Disease Self Management

- Basic training in nutrition (to target Primary Care Providers and Nurse Care Managers)

- "What works" sessions for sharing best practices across clinics and throughout the hospital system

- Licensing guidelines in relation to team roles (e.g., what is an LPN allowed by law to do in this state, and what is the LPN expected to do as part of the team?)

- Computer skills for appropriate patient scheduling

- Practical rotations for all employees (but prioritized for Clinical and Clerical Associates) outside of Primary Care, to understand integrated hospital structure

- In-depth module on the Patient Centered Medical Home to be developed for new employee orientation

Originally, VA disseminated PACT training by providing one facility team with intensive training over several workshops; that team then disseminated knowledge through presentations to other teams. This model was widely seen as insufficient, and more direct structured training was desired. VA's subsequent attempts to provide training to all teams were cut short by budget limitations. Several participants stressed the importance of having "all of us trained equally" 
and felt the current situation created a "disparity in knowledge" that made it difficult to implement clinic-wide changes across teams with different skill levels.

\section{Theme 5: Facility and Clinic Leadership Must Champion Employees and Model}

Where PACT implementation was felt to be going well, employees often described the importance of clinic and facility level management acting as "champions" for employees and for the model itself. Text Box 2 lists ways that managers were seen to demonstrate this commitment. In clinics associated with one hospital facility, many employees felt progress had occurred because Primary Care Division leadership had successfully advocated for funding additional positions despite a federal government hiring freeze. Perceptions of facility (not clinic) leadership in this facility's clinics were more positive than in the clinics in other participating facilities. At the clinic level, employees felt PACT was aided by leadership that encouraged and supported all employees, not just PCPs.

One of the things I like about this clinic is that I think I'm allowed to do almost anything that I would want to do. I have a manager that if I want to add on group visits he's very positive and would let you do that. ...they let me work to the highest level of my job description. I like that.... I know, talking to some of the nurses in other CBOC's, they don't have a lot of the freedoms that I do. [NCM]

\section{Text Box 2. Actions that demonstrate clinic leaders/management are committed to the Patient Centered Medical Home (PCMH) model of care}

- Allocate time for staff to attend trainings about PCMH: what it is and how to implement it

- Advocate for and defend PCMH innovations to facility leadership (e.g., authorize clinic time for population management or telephone clinic; press for greater staffing or expanded service offerings)

- Organize team training at the clinic level (hold clinic retreats to discuss team roles; use the Meyers-Briggs psychometric questionnaire to facilitate discussion of team dynamics)

- Model a team approach by involving all employees in opportunities for education, development of clinic initiatives, etc.

- Define team member roles (for example, make the development of PCMH-aligned written job descriptions a priority, and allocate time to discuss roles in clinic meetings)

- Make and communicate clear policies about how PCMH changes the flow of clinic activities (for example, who should answer phones to promote team function)

- Encourage teams to try PCMH components, like group visits, that are new to them

- Promote a clinic culture that allows experimentation (and failure)

In contrast, at clinics where implementation was not felt to be going well, management was described as unresponsive and unaligned with PACT goals:

[Manager is] very interested in... control. Never asks, does this help us take care of the vet? No? Is this a rule... being followed to the nth degree? That's the only question that's asked. [PCP]

I think I don't trust my management anymore to respond to us.... It is very adversarial with our local leadership. [PCP]

\section{OBSERVATIONS ON SIMILARITIES AND DIFFERENCES RELATED TO CLINIC AND PARTICIPANT CHARACTERISTICS}

Our study purposefully included participants from a variety of professions and roles, and clinics with a wide range of characteristics. Though a detailed comparison of participant responses is outside the scope of this paper, some interesting patterns emerged. Clinics with high turnover or frequent use of locums often described lack of continuity as a foundational challenge for implementation. An academic 
teaching clinic with many part-time providers faced unique difficulties in implementing a model that assumes one provider per panel. Rural clinic employees often emphasized transportation and other barriers for patients who required services only available at urban VA hospitals. Employees were thus quick to identify what they saw as their clinic's unique characteristics, but always with reference to the common themes of teams, adequate staffing and integration of PACT practices into the VA system that were shared across clinics.

We observed some differences in the experience of PACT influenced by a team member's role. Clerical and Clinical Associates most often felt that PACT had actually changed what they did and felt stimulated by expanded responsibilities, even in the face of increased work volume. In contrast, Nurse Care Managers more often described PACT implementation as an unfulfilled opportunity. While in theory they were able (and expected) to do more "top of license" work, stress resulted from not having enough time to do it. Primary Care Providers often felt PACT created new performance expectations for data collection and management, without any additional time or resources being allocated to meet these expectations. At the same time, many providers, even those who criticized current practices, felt a strong alignment with the model and hoped for its success:

This is certainly the closest I've ever been to working on an actual medical home model that functions. I think we're in a good place to go from there. It's a sincere drive. I've been quite hopeful about it, actually. [PCP]

\section{DISCUSSION}

Our qualitative study found that primary care employees in a variety of roles believe in the promise of team-based care, but have been challenged by the realities of implementation. Participants identified prerequisites to successful PACT implementation, including increased staffing, changes in VA system practices, team development training, and leadership that demonstrates commitment to PACT values. In contrast to many clinics that implement electronic health record (EHR) use as part of a PCMH transition, the VA has used EHRs for patient care and data-driven improvement for over a decade. Despite this facilitating context, human challenges to make use of these data tools clearly remained, with the root cause of difficulties going back to inadequate staffing or unclear team roles. This suggests that practices implementing PCMH must pay at least equal attention to human and technological resources. Our findings align well with the importance of "change process capability" emphasized in Solberg's model of medical practice change. ${ }^{14}$
While VA leadership prioritized the concept of PACT (the first, necessary step towards change in that conceptual model), and EHRs were already in place, clinical capacity to adopt new practices was felt to be limited by systemically enforced constraints and the lack of team member skills to manage change and work as teams.

Recent PCMH literature emphasizes the profound cultural shift involved in moving from physician-led to team-based and patient-centered care. ${ }^{11,15-17}$ Our findings align with Howard and colleagues ${ }^{18}$ emphasis on the importance of inclusive leadership. While others ${ }^{19}$ have examined leadership through interviews with clinic management, our study confirms that the "led"- the clinic employees - keenly feel the difference good leadership makes. In the context of primary care clinics operating within large integrated health systems, an equal challenge lies at the level of changing institutional culture and practice. Our findings add to research ${ }^{20}$ emphasizing that institutional commitment to the PCMH model is needed to make necessary systemic alignments, in everything from allocation of funds to hiring practices. This includes developing financial reimbursement mechanisms that will capture and reward the work done by teams to manage populations proactively. In VA, as in other large provider networks, teams must function within a structure, which, by necessity, controls allocation of resources and values standardization of care processes. Pilot sites explicitly fostering greater local control could be charged with developing best practices for balancing local-central priorities and could inform the continuing evolution of PACT in practice.

Our participants' experiences led them to independently generate a definition of a well-functioning team that strikingly mirrors the observations of a recent review of successful PCMH projects, which identified data-driven improvement, panel management, and team-based care as the three essential "building blocks" of the PCMH approach. ${ }^{3}$ As others have also found, ${ }^{21,22}$ the skills for effective team-based care are distinct from clinical skills and need to be taught.

The recommended team staffing model proposed by VA differs from that in other PCMH models, which do not pair every PCP with a dedicated full-time $\mathrm{NCM}^{23}$ We found that most employees in all roles felt that PACT's recommended four-person team structure did work well, once in place. Our study's finding that Clinical and Clerical Associates in particular found increased job satisfaction within team-based care is interesting, in light of Day and colleagues ${ }^{\circ 8}$ finding that continuity and engagement in the Medical Assistant role was associated with several positive PCMH outcomes. Similarly, Gabbay and colleagues ${ }^{24}$ found that an expanded medical assistant role was characteristic of higher performing PCMH practices. These findings collectively suggest that the presence of high performing "support" or "associate" employees may in fact be central to PCMH success. Since the use of team-based 
care is often put forward as one piece of the solution to shortages in the US primary care provider workforce, maximizing the model's potential to create professional satisfaction for all team members is a practical necessity.

Practice change is increasingly accepted as difficult and ongoing, requiring years to show results..$^{9,17,20,25,26} \mathrm{~A}$ survey of medical home implementation in 65 safety-net clinics found that greater implementation of characteristics of the medical home was associated with both higher morale and higher provider burnout. ${ }^{27}$ This finding is consistent with the context we encountered, where many participants described PACT implementation as both exciting and stressful. PACT offered new opportunities (which could improve morale), while also bringing the barriers into stark relief (which could increase burnout). Trade-offs between different outcome measures and staff satisfaction have been found in other systems implementing PCMH models. ${ }^{7}$ Monitoring and addressing burnout will be important throughout PACT implementation.

Limitations. Our study was limited to VA clinics within one region. The VA's integrated healthcare system, with salaried physicians and a large but clearly defined veteran client population, differs in many ways from other healthcare systems implementing PCMH models. Our clinic data were collected on an opportunistic 22 month time line, with participating clinics at different points in implementation at the time we conducted focus groups/interviews; this limits our ability to make comparisons across their experiences. As in many early PCMH efforts, ${ }^{21}$ patient involvement with practice transformation was extremely limited in our study clinics. We limited our focus to the perceptions of employees; interviewing patients during this time of transition in care practice might have yielded a different picture of key ingredients for PACT success. As with any qualitative study, our methods were chosen to obtain a rich, in-depth understanding of a particular context, and our findings have limited generalizability. At the same time, a strength of our study was its large interdisciplinary sample that included all primary care clinic roles, from a range of VA clinics. Our results describe in depth the experience of implementation among front-line clinical employees, identifying both shared experiences and unique clinic contexts.

Implications. VA primary care employees have identified several potential areas for improvement in the VA's ongoing implementation of a PCMH model. These include the training of all employees in conceptual and practical skills related to team function, and changes in hiring and orientation practices, such as hiring complete teams together and offering pragmatic PACT skills training in orientation, to more directly support the development of well-functioning teams. Clinics implementing a PCMH model of care should focus early on effective teambuilding, since challenges to human capacity for change are likely to exist even when, as in the VA, integrated data and financial reimbursement systems are already in place. As the VA enters the fifth year of its PCMH implementation, moving forward with PACT will require continued change not just within primary care clinics, but in the hospital, specialty, and administrative systems within which they are integrated.

Acknowledgements: We gratefully acknowledge the contribution of all members of the VISN 20 PACT Demonstration Lab, as well as the employees in participating clinics, who generously shared their experiences. Funding for the PACT Demonstration Laboratory initiative is provided by the VA Office of Patient Care Services. Dr. Tuepker is a Health Science Specialist at the Portland VA Medical Center. The views expressed in this article are those of the authors and do not necessarily represent the views of the Department of Veterans Affairs.

Conflict of Interest: The authors declare that they do not have a conflict of interest.

Corresponding Author: Anaïs Tuepker, PhD, MPH; Portland VA Medical Center, Health Services Research Development/ VISN 20 PACT Demonstration Laboratory, Veterans Health Administration, Mail code RD63, PO Box 1034, Portland, OR 97239, USA (e-mail: anais.tuepker@va.gov).

\section{REFERENCES}

1. Cassidy A. Patient-centered medical homes. Health Aff 2010; 29:1-6.

2. Bodenheimer T, Yoshio Lang B. The teamlet model of primary care. Ann Fam Med 2007; 5:457-61.

3. Willard R, Bodenheimer T. The Building Blocks of High Performing Primary Care: Lessons from the Field. 2012. California HealthCare Foundation.

4. Williams JW, Jackson G, Powers B, et al. The Patient Centered Medical Home Closing the Quality Gap: Revisiting the State of the Science. 2012. Rockville, MD: Agency for Healthcare Research and Quality.

5. Yoon J, Rose DE, Canelo I, et al. Medical home features of VHA primary care clinics and avoidable hospitalizations. J Gen Intern Med 2013: doi: 10.1007/s11606-013-2405-5

6. Reid RJ, Johnson EA, Hsu C, et al. Spreading a medical home redesign: effects on emergency department use and hospital admissions. Ann Fam Med 2013; 11 (S1):S19-26.

7. Driscoll DL, Hiratsuka V, Johnston JM, et al. Process and outcomes of patient-centered medical care with Alaska Native people at Southcentral Foundation. Ann Fam Med 2013; 11(S1):S41-49.

8. Day J, Scammon DL, Kim J, et al. Quality, satisfaction, and financial efficiency associated with elements of primary care practice transformation: preliminary findings. Ann Fam Med 2013; 11(S1): S50-59.

9. McNellis RJ, Genevro JL, Meyers DS. Lessons learned from the study of primary care transformation. Ann Fam Med 2013; 11(S1): S1-5.

10. Tomoaia-Cotisel A, Scammon DL, Waitzman NJ, et al. Context matters: the experience of 14 research teams in systematically reporting contextual factors important for practice change. Ann Fam Med 2013; 11(S1):S115-123.

11. Cronholm PF, Shea JA, Werner RM, Miller-Day M, Tufano J, Crabtree BF, Gabbay R. The patient centered medical home: mental models and practice culture driving the transformation process. J Gen Intern Med 2013; 28(9):1195-201.

12. Damschroder LJ, Aron DC, Keith RE, et al. Fostering implementation of health services research findings into practice: a consolidated framework for advancing implementation science. Implementation Science 2009; 4(50) doi:10.1186/1748-5908-4-50.

13. Hsieh HF, Shannon SE. Three approaches to qualitative content analysis. Qual Health Res. 2005; 15 (9): 1277-88.

14. Solberg LI. Improving medical practice: a conceptual framework. Ann Fam Med 2007; 5 (3): 251-56. 
15. Ghorob A, Bodenheimer T. Sharing the care to improve access to primary care. N Engl J Med. 2012;366(21):1955-1957.

16. Nutting PA, Miller WL, Crabtree BF, et al. Initial lessons from the first national demonstration project on practice transformation to a patientcentered medical home. Ann Fam Med. 2009; 7(3): 254-260.

17. Markova T, Mateo M, Roth, LM. Implementing teams in a patientcentered medical home residency practice: lessons learned. J Am Board Fam Med. 2012; 25 (2); 224-31.

18. Howard J, Shaw EK, Felsen CB, et al. Physicians as inclusive leaders: insights from a participatory quality improvement intervention. $\mathrm{Q}$ Manage Health Care 2012; 21(3): 135-45.

19. McMullen CK, Schneider J, Firemark A, et al. Cultivating engaged leadership through a learning collaborative: lessons from Primary Care Renewal in Oregon Safety Net Clinics. Ann Fam Med 2013; 11(S1):S34-40.

20. Solimeo SL, Hein M, Paez M, Ono S, Lampman M, Stewart GL. Medical homes require more than an EMR and aligned incentives. Am J Manag Care. 2013; 19(2): 132-40.

21. Wynia MK, Von Kohorn I, Mitchell PH. Challenges at the intersection of team-based and patient-centered health care: insights from an IOM working group. JAMA 2012; 308 (13): 1327-28.
22. Scholle SH, Asche SE, Morton S, Solberg LI, Tirodkar MA, Jaén CR. Support and strategies for change among small patient-centered medical home practices. Ann. Fam. Med. 2013; 11 (S1): S6-13.

23. Hsu C, Coleman K, Ross TR, Johnson E, Fishman PA, Larson EB, Liss D, Trescott C, Reid RJ. Spreading a patient-centered medical home redesign: a case study. J Amb Care Manage. 2012: 35(2); 99-108.

24. Gabbay RA, Friedberg MW, Miller-Day M, Cronholm PF, Adelman A, Schneider EC. A positive deviance approach to understanding key features to improving diabetes care in the medical home. Ann Fam Med 2013; 11 (S1): S99-107.

25. Howard J, White $\mathbf{P}$, Chronister $\mathbf{K}$, Balamurugan $\mathbf{A}$. Bringing home the patient centered medical home: lessons learned from an academic family medical center. J Arkansas Med Soc. 2012: 108 (12); 300-03.

26. Solberg LI, Crain AL, Tillema J, et al. Medical home transformation: a gradual process and a continuum of attainment. Ann. Fam. Med. 2013: 11 (S1): S108-114.

27. Lewis SE, Nocon RS, Tang $\mathbf{H}$, et al. Patient-centered medical home characteristics and staff morale in safety net clinics. Arch Intern Med. 2012; 172(1):23-31. 